Homology, Homotopy and Applications, vol.21(2), 2019, pp.27-28

\title{
STABLE INDECOMPOSABILITY OF THREE-MANIFOLDS
}

\author{
M. J. D. HAMILTON AND D. KOTSCHICK
}

(communicated by Donald M. Davis)

\begin{abstract}
We explain that a variant of a recent result of KwasikSchultz $[\mathbf{3}]$ about stable indecomposability of three-manifolds is an immediate consequence of results of Kotschick, Löh and Neofytidis $[\mathbf{2}, \mathbf{1}]$.
\end{abstract}

The following result was proved recently by Kwasik and Schultz; see [3, Theorem A]:

Theorem 1.1. Let $M$ be a closed oriented 3-manifold that is not a Cartesian product. Then there is no closed oriented manifold $N$ of dimension $\leqslant 3$ such that $M \times N$ decomposes as a Cartesian product of surfaces and the circle.

We show that the following variation on the theme of this theorem follows directly from the results of Kotschick, Löh and Neofytidis in $[\mathbf{2}, \mathbf{1}]$ :

Theorem 1.2. Let $M$ be a closed oriented 3-manifold that is not finitely covered by a Cartesian product. Then there is no closed oriented manifold $N$, of any dimension, such that $M \times N$ decomposes as a Cartesian product of surfaces and the circle.

This is both weaker and stronger than Theorem 1.1. It is weaker in that we not only assume that $M$ is not a product, but make the stronger assumption that $M$ is not finitely covered by a product. It is stronger in that it does not require the assumption $\operatorname{dim}(N) \leqslant 3$.

Proof of Theorem 1.2. Recall that a closed oriented 3-manifold is rationally essential if and only if it has an aspherical summand in its Kneser-Milnor decomposition; cf. [2, Theorem 3]. With the additional assumption of rational essentialness one has the following much stronger conclusion than in Theorems 1.1 and 1.2:

Proposition 1.3. Let $M$ be a rationally essential closed oriented 3-manifold that is not finitely covered by a Cartesian product. Then there is no closed oriented manifold $N$ such that $M \times N$ admits a non-zero degree map from a Cartesian product of surfaces and the circle.

Proof. Since $M$ is rationally essential and not finitely covered by a product, it is not dominated by a product by [2, Proposition 1]. Therefore, the conclusion follows from $[\mathbf{1}$, Theorem 2.3].

Received July 19, 2018; published on December 19, 2018.

2010 Mathematics Subject Classification: 57N65.

Key words and phrases: Cartesian product, domination, three-manifold.

Article available at http://dx.doi.org/10.4310/HHA.2019.v21.n2.a3

Copyright (c) 2018, M. J. D. Hamilton and D. Kotschick. Permission to copy for private use granted. 
Most cases of Theorem 1.2 follow from Proposition 1.3. The missing cases concern the rationally inessential manifolds that are not finitely covered by products. By $[\mathbf{2}$, Theorem 3], a rationally inessential $M$ is finitely covered by some $\#_{k}\left(S^{1} \times S^{2}\right)$ for $k \geqslant 0$. Moreover, the case $k=1$ is excluded by the assumption that $M$ is not finitely covered by a product.

If $k \geqslant 2$, then $\pi_{2}(M)=H_{2}(\widetilde{M} ; \mathbb{Z})$ is not finitely generated since $\widetilde{M}$ is the universal covering of $\#_{k}\left(S^{1} \times S^{2}\right)$. For such $M$ the conclusion of Theorem 1.2 follows from the fact that for a product of closed orientable surfaces and the circle, the second homotopy group is finitely generated, with generators the $S^{2}$-factors in the product.

Finally, if $k=0$, then $M$ is finitely covered by $S^{3}$ and the universal covering of any $M \times N$ splits as $S^{3} \times \widetilde{N}$. In particular, $H_{3}(\widetilde{M \times N} ; \mathbb{Z}) \neq 0$. For such an $M$ the conclusion of Theorem 1.2 follows from the fact that for a product of closed orientable surfaces and the circle, the universal covering is a product of two-spheres and Euclidean spaces; in particular its third homology vanishes.

This completes the proof of Theorem 1.2.

\section{References}

[1] D. Kotschick, C. Löh and C. Neofytidis, On stability of non-domination under taking products, Proc. Amer. Math. Soc. 144 (2016), 2705-2710.

[2] D. Kotschick and C. Neofytidis, On three-manifolds dominated by circle bundles, Math. Z. 274 (2013), 21-32.

[3] S. Kwasik and R. Schultz, Decomposing manifolds into Cartesian products, Homology, Homotopy Appl. 20 (2018), no. 2, 1-10.

M. J. D. Hamilton mark.hamilton@math.lmu.de

Institut für Geometrie und Topologie, Universität Stuttgart, Pfaffenwaldring 57, 70569 Stuttgart, Germany

D. Kotschick dieter@math.lmu.de

Mathematisches Institut, LMU München, Theresienstr. 39, 80333 München, Germany 